\title{
Effect of Ablation on Local Activation Intervals and Dominant Frequencies of Fibrillation
}

\author{
SM Abashian, AC Kiser, HD Himel, JH Dumas, SB Knisley \\ The University of North Carolina at Chapel Hill, Chapel Hill, NC, USA
}

\begin{abstract}
Activation frequencies of tachyarrhythmias may guide production of high-quality ablation lesions. To examine this in rabbit ventricles, frequencies from bipolar potentials adjacent to a continuous and transmural lesion were analyzed with a time-domain algorithm and FFT. Time-domain analysis produced greater variability among frequencies compared with dominant frequencies from the FFT. With either timedomain or FFT, frequency distributions were different after vs. before ablation. Means of frequencies had equal probability to increase or decrease, while standard deviations decreased after production of a continuous and transmural lesion. Thus, both the time-domain method and FFTs indicate reduced variation of activation frequencies by ablation.
\end{abstract}

\section{Introduction}

Measures of frequency of excitation during atrial fibrillation or ventricular tachyarrhythmia are informative metrics of the arrhythmias. [1-3] An intraoperative diagnosis that uses frequency to indicate whether an individual lesion is continuous and transmural may help operators produce high-quality lesions during fibrillation. Methods that use pacing to diagnose lesion quality are not normally applicable during fibrillation. [4] A diagnosis that uses frequency would require rapid determination of the frequency. Also the accuracy of the measurement may depend on whether the frequency is determined with spectral methods in the frequency domain or with individual beat-to-beat intervals in the time domain. [5]

The Fast Fourier Transform (FFT) requires a certain electrogram segment to determine dominant frequency, and does not report on individual intervals. To determine frequency information in a shorter time and for individual intervals, we developed an algorithm that estimates frequencies in the time domain and is suitable for realtime programming. Here we demonstrate its ability to determine frequency content of fibrillation before and after ablation, and compare results with the corresponding results obtained using the FFT method.

\section{Methods}

Fibrillation was induced in 10 isolated perfused rabbit hearts by pacing at increasingly fast rates using 10 ms decrements of the pacing interval until fibrillation was observed upon turning off the stimulus. A linear suction radiofrequency (RF) ablation or coagulation probe (nContact, Surgical, Morrisville, NC) having $2.5 \mathrm{~cm}$ length was placed on the left ventricular epicardium. Extracellular potentials were digitized at a rate of $10 \mathrm{KHz}$ from the epicardium using separate bipolar electrode pairs located on each side of the probe.

For analysis in the time domain, times of beats were defined from maxima of bipolar potentials. Frequencies were determined from reciprocals of beat-to-beat intervals. For analysis in the frequency domain, the recordings were divided into consecutive 2 s segments. The spectrum was computed as the product of the complex FFT and its complex conjugate. Dominant frequencies were determined for each segment.

Examples of the bipolar potentials that were recorded during fibrillation and the frequency spectra obtained from the recordings are shown in figures 1 and 2.

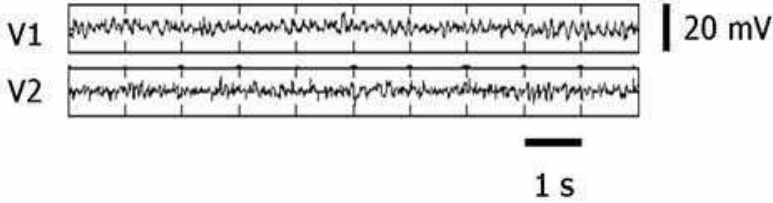

Figure 1. Bipolar potentials recorded from each side of $\mathrm{RF}$ probe (V1 and V2) during fibrillation. Intervals between consecutive beats were determined for all local maxima exceeding a cutoff value of $1 / 4$ of the greatest potential found in $10 \mathrm{~s}$. Reciprocals of the intervals were used as estimates of frequency. 


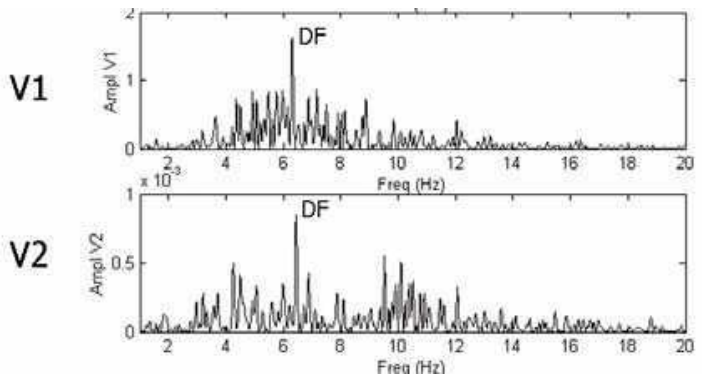

Figure 2. Frequency spectra of $2 \mathrm{~s}$ segments of bipolar potentials from each side of RF probe during fibrillation. Dominant frequency was the frequency between 1 and 20 $\mathrm{Hz}$ at which the spectrum had the greatest amplitude.

\section{Results}

Figure 3 shows photographs of a heart after postexperimental perfusion with a stain to highlight viable tissue. The ablated tissue appears lighter than the viable tissue. Examination of the stained hearts showed that lesions produced by the RF ablation were continuous and transmural.
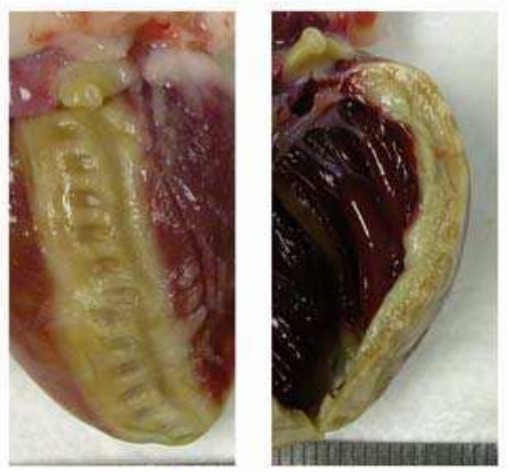

Figure 3. Epicardial view (left) and transmural section (right) of continuous transmural lesion in left ventricle of rabbit heart. Postexperimental perfusion with a tetrazolium chloride solution stained the viable tissue red. Lesion extended from apex of heart to atrio-ventricular groove.
The time-domain analysis provided more frequencies for a given segment length, e.g., 5-10 measurements of frequency per second of electrogram segment. This is due to the high rate of excitation during fibrillation, and the fact that the time-domain algorithm may detect the individual intervals between consecutive excitations. The FFT method used a 2-second electrogram to obtain each dominant frequency.
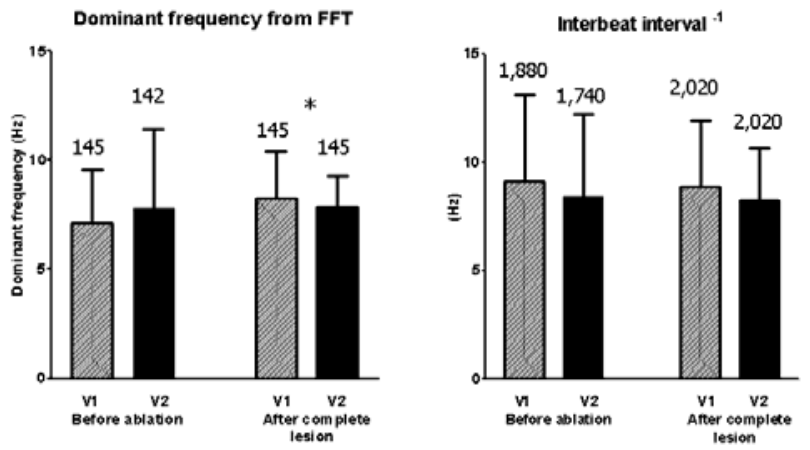

Figure 4. Frequencies during $290 \mathrm{~s}$ of fibrillation in 10 hearts. Dominant frequencies before and after ablation are shown on the left plot. The frequencies estimated from the interbeat intervals are shown on the right. Same recordings were used in both analyses.

* indicates $\mathrm{p}<0.05$ from F-test for standard deviation after ablation compared to value before ablation (For dominant frequency, $\mathrm{n}=287$ before ablation and 290 after ablation. For interbeat interval, $n=3,620$ before ablation and 4,040 after ablation. Numbers of measurements from each side of RF probe are indicated above bars.)

With either time-domain or FFT, distributions of frequencies were different after vs. before ablation. Means of frequencies had equal probability to increase or decrease, while standard deviations decreased after production of a continuous and transmural lesion.

\section{Discussion and conclusions}

The results indicate ablation decreases the standard deviation of frequencies of fibrillation. This may result from an increase in organization of the arrhythmia, producing frequencies that are more similar. While this might be expected with elimination of a reentrant circuit by ablation, a full explanation will probably require cardiac mapping.

The standard deviations were greater for the timedomain analysis vs. the FFT (Figure 4). This suggests variability of intervals is not fully represented in the dominant frequencies. [5] 
Magnitudes of maxima of bipolar potentials produced by excitations varied substantially during fibrillation (Figure 1). The reason for this is probably multifactorial, and may depend on the angle of the excitation front in the measurement region and the excitability state of the cells. Cells in fibrillation can undergo nonuniform conduction or block, which affect potentials. In order to identify excitations, the time-domain algorithm used a cutoff value of $1 / 4$ of the greatest potential. Lowering the cutoff produces an increase in the number of excitations detected. [4] Although the optimal cutoff is unknown, the value we used produced mean frequencies similar to those from the FFT.

\section{Acknowledgements}

This research was supported by nContact Surgical, Inc, Morrisville, NC, and the Department of Biomedical Engineering of The University of North Carolina at Chapel Hill and North Carolina State University.

\section{References}

[1] Sanders P, Berenfeld O, Hocini M, Jaïs P, Vaidyanathan R, Hsu L-F, et al. Spectral analysis identifies sites of highfrequency activity maintaining atrial fibrillation in humans. Circ 2005;112(6):789-797.

[2] O’Neill MD, Jaïs P, Takahashi Y, Jönsson A, Sacher F, Hocini M, et al. The stepwise ablation approach for chronic atrial fibrillation-Evidence for a cumulative effect. J Int Cardiac Electrophysiol 2006;16(3):153-167.

[3] Lazar S, Dixit S, Callans DJ, Lin D, Marchlinski FE, Gerstenfeld EP. Effect of pulmonary vein isolation on the left-to-right atrial dominant frequency gradient in human atrial fibrillation. Heart Rhythm 2006:889-895.

[4] Himel HD, Dumas JH, Kiser AC, Knisley SB. Translesion stimulus-excitation delay indicates quality of linear lesions produced by radiofrequrency ablation in rabbit hearts. Physiological Measurement 2007;28:611-623.

[5] Ng J, Kadish AH, Goldberger JJ. Effect of electrogram characteristics on the relationship of dominant frequency to activation rate in atrial fibrillation. Heart Rhythm 2006;3:1295-1305.

Address for correspondence

Stephen B. Knisley

Department of Biomedical Engineering 152 MacNider Hall

Chapel Hill, NC 27599-7575 USA 